\title{
Modeling the Deformation of the Aerodynamic Surface to Estimate the Error of the Photogrammetric Algorithm*
}

\author{
Anton Poroykov1 [0000-0002-9284-1397], Nataliya Ilina1 [0000-0002-1793-4606] \\ and Ekaterina Shmatko ${ }^{1}$ [0000-0003-3565-9502] \\ National Research University "Moscow Power Engineering Institute", \\ Krasnokazarmennaya 14, Moscow, 111250, Russia \\ poroykovay@gmail. com \\ nataliya48452@gmail.com \\ shmatko.97@bk.ru
}

\begin{abstract}
In-flight measurements are one of the directions of aircraft testing which has been developed actively recently. Creation of a flight laboratory based on an unmanned vehicle to reduce the cost of developing and analysis techniques for in-flight measurements is proposed. For the laboratory an onboard system for recording experimental images during a flight was developed. It was decided to conduct computer modeling of images that can be obtained using the developed system for its verification. Unity a cross-platform photorealistic graphic engine with visual integrated development environment was chosen for this purpose. For modeling both external and internal real camera parameters, including distortion coefficients, and parameters of the measured surface could be preassigned. The developed methodology for computer modeling of images of the deformable surface allows to evaluate the accuracy of various algorithms for processing experimental images, as well as the influence of the parameters of the experimental setup on the error of the measurements results.
\end{abstract}

Keywords: Computer Modeling, Shape Measurement, Measurement Errors Image Pattern, Correlation Technique.

\section{Introduction}

In-flight measurements are one of the directions of aircraft testing which has been developed actively recently. These measurements provide the ability to measure various parameters directly in flight [1]. The development of these type of methods and techniques is carried out by the international scientific and technical collaborations, for example AIM and AIM2 [2]. Examples of practical tests include in-flight tests of the

Copyright $(2020$ for this paper by its authors. Use permitted under Creative Commons License Attribution 4.0 International (CC BY 4.0).

Publication financially supported by RFBR grant №20-01-00547 
VUT100 Cobra [3], Airbus A320 [4] and even Airbus A380 [5]. The practical implementation of this approach allows to accelerate both the development of new types of aircraft, and the in-flight tests of serial samples, and their commissioning.

Carrying out such tests is associated with high cost. Therefore, the creation of a flight laboratory, based on an unmanned vehicle, was proposed [6]. On the one hand, this will significantly reduce the cost of testing, and on the other, the test conditions will be close to real in-flight tests: an increased level of vibration, the influence of aerodynamic loads and flight parameters on the deformation of the airframe, strong exposure to the Sun. The application of this approach will allow to improve existing and develop new methods and techniques for in-flight tests.

The first method to be studied at the proposed laboratory will be the method of measuring deformations (surface shape) - Image Pattern Correlation Technique (IPCT) [78]. It is based on digital processing of stereo images to restore an object surface shape. Processing is based on correlation methods that are widely used in flow diagnostic methods: PIV (Particle Image Velocimetry) [9] and BOS (Background Oriented Schlieren) [10]. For this purpose, an electronic optical system for recording stereo images in flight was developed. A drone wing was selected as the object of the study. The system allows to receive images on the aircraft board and transmit them wirelessly to the ground to the operator's computer. The system also allows to obtain flight parameters to estimate their impact on the magnitude and type of deformations.

To conduct bench tests of the developed system, it was proposed to use the previously created experimental setup for estimating the error of photogrammetric methods [11-12]. However, due to a number of circumstances, laboratory testing was not possible. Therefore, it was decided to conduct computer modeling of images that can be obtained using the developed system.

The main idea of modeling is to set the shape of the surface that simulate the wing during deformation, and to obtain its images with changes caused by this deformation. After processing the images and restoring the surface shape using the IPCT, this result can be compared with the wing surface coordinates predefined in the modeling. The deviation between the points of the modeled and the calculated surfaces can serve as an estimate of the error of the developed system and the selected processing algorithm. By changing the modeling parameters: the position of the camera stereo system, the background pattern parameters and the deformation amplitude, experimental dependences can be obtained to estimate the measurement error for various parameters of the inflight experiment.

\section{Surface modeling technique}

A cross-platform environment for the development of computer games - Unity [12] was chosen as the basis for modeling. Its main advantages are the presence of a visual integrated development environment, a photorealistic graphics engine, support for solid state physics and the ability to write scripts in $\mathrm{C \#}$. The latter fact allows to use all the features of the language in the modeling and connect additional libraries. 
In computer graphics, the smallest flat figure is a triangle and all complex surfaces are broken into them. This is due to the fact that the surface of the triangle remains flat during its points moving. Therefore, to simulate the deformation of the wing surface, it is necessary to break it into triangles. Moreover, one of the assumptions used for modeling is the absence of elastic deformations of the surface. In other words, the area of the wing surface remains constant under all deformations. Therefore, the sides of the triangle should not change.

To implement such triangles, one of the types of objects built into Unity - rigid bodies can be used. These objects do not change their shape over time, while they are taken into account in the work of the physical engine. They can be affected by gravity, forces from other rigid bodies, and their speed and acceleration will be determined by the magnitude, direction and time of application of the resultant force. Rigid bodies in Unity can be interconnected using joints that simulate connections. This method was chosen to create the surface of the simulated wing.

At the beginning of the modeling, the size of the wing and the number of partitions of its surface into triangles vertically and horizontally are set. The wing is defined by a rectangular surface. It, in turn, is divided into rectangles, and then each rectangle into two triangles (see Fig. 1a). Neighboring triangles are connected using joints at two angles (see Fig. 1b). Four triangles in the corners of the surface are fixed in space on soft links to maintain the surface in a horizontal position. The texture of the background pattern is applied to the surface. When moving a triangle, the corresponding part of the texture of the background screen moves with it.

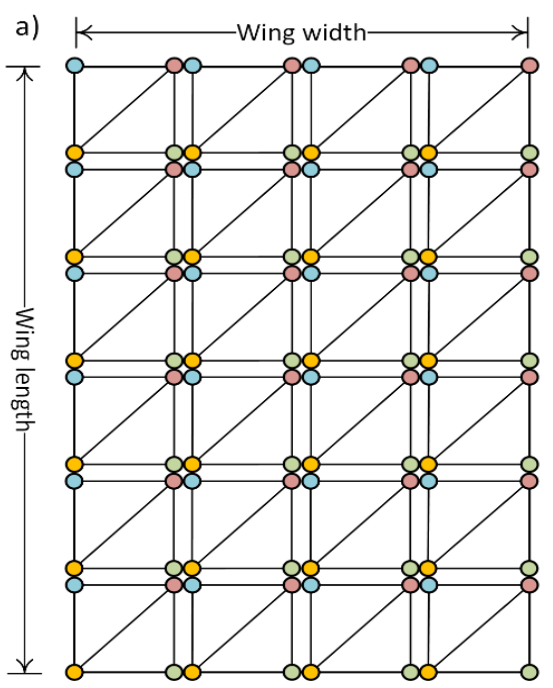

b)

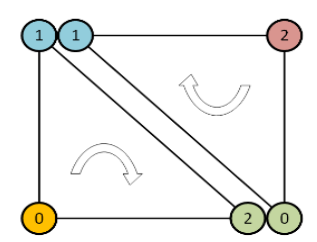

c)

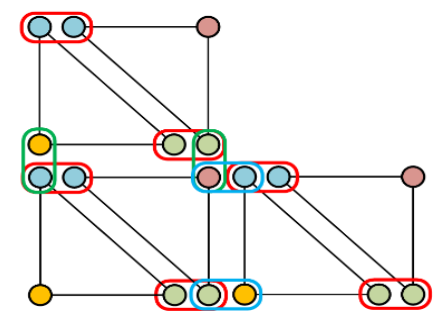

Fig. 1. The scheme of formation of the wing surface from triangles and their connection: a) the partition of the wing surface into triangles, b) the formation of two adjacent triangles of four vertices, c) horizontal and vertical connections between the vertices of the triangles. 
To specify the surface, the coordinates of the vertices of the rectangles are first calculated. The vertex coordinates are stored in a separate Verticies property of the object, which is the wing surface in Unity. Next, each rectangle is divided into two triangles. To record the vertices of triangles, not their coordinates are indicated, but the coordinate numbers in the Verticies property. Therefore, four vertices are used to define two triangles, not six (see Fig. 1b). Coordinate numbers are stored in the Triangles property. The vertices must be specified in clockwise order. This condition defines the side on which the texture will be displayed.

Each triangle is created as a rigid body. To simulate surface deformation, triangles are interconnected. Two triangles in one rectangle are interconnected using two horizontal joints (see Fig. 1c) having coordinates that coincide with the coordinates of two common vertices of the triangles. The adjacent horizontal rectangles are interconnected using two joints, the coordinates of which coincide with the coordinates of two common vertices of the two outside triangles. Neighboring rectangles vertically are connected using two joints, the coordinates of which coincide with the coordinates of two common vertices of four triangles (see Fig. 1c).

Thus, the simulated wing surface consists of a large number of interconnected rigid bodies, which are triangles. Their joints have a large number of settings, with the help of which the interaction of the triangles: the limiting angles of rotation in two axes, the forces acting when they are exceeded, etc., are configured. This allows to set the rigidity of the simulated wing. If necessary, force can be applied to individual triangles with a given direction and magnitude. This allow simulating deformations caused by aerodynamic loads.

The software for modeling is a compiled program for the 32-bit or 64-bit Windows operating system (it is possible to create executable files for other platforms). The view of the graphical user interface of the program is presented in Fig. 2.

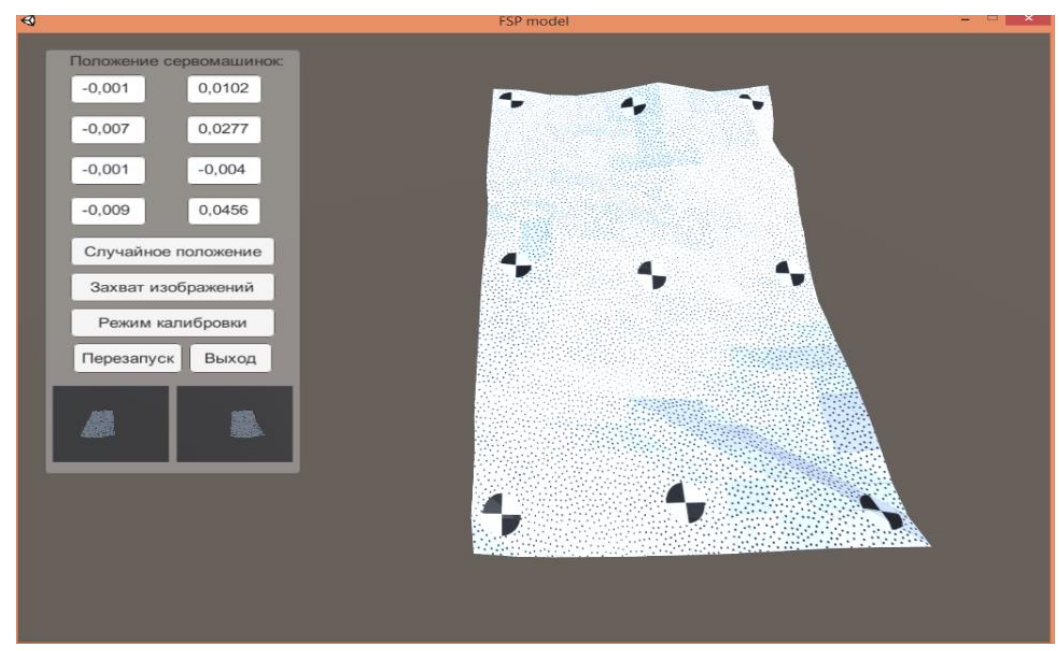

Fig. 2. Graphical interface of the program for modeling wing surface deformation. 
The control panel is located on the left side of the window. There are buttons on it for setting a random position of the surface, for capturing images from the camera stereo system, turning on the calibration mode, restarting the modeling and exiting the program. In the window itself, the view of the wing is presented from the third camera, which is not included in the stereo system. It is located at a large angle to the surface for visual inspection. Two small windows are situated at the bottom of the panel. They display the image from the cameras included in the stereo system. The text fields above the buttons in the control panel are for indicating and setting the positions of eight triangles on the surface. These triangles simulate the operation of servos in a laboratory setup [11-12].

On click on the "Image Capture" button, the images received from the stereo system cameras are saved to the folder with the modeling program. At the same time, a json file is saved with the coordinates of the triangles. These coordinates are the exact value of the position of the surface and can serve to estimate the accuracy of the results obtained by restoring the shape of the surface during image processing.

To obtain calibration data of the stereo system, a calibration mode is provided. In this mode, the wing surface is not displayed, but instead a calibration target appears with the structure of a chessboard. The target randomly changes its position in space to simulate the calibration procedure on a real setup. In each position, images are captured from the stereo system, which are then used to calculate the external and internal parameters of the cameras, as well as their relative position in stereo system.

\section{$3 \quad$ Modeling results and their processing}

The scheme for reconstructing the surface shape using the IPCT which was used in the work consists of:

- application of a background pattern on the surface under study (on the model of the deformable wing);

- registering images of the background pattern from two cameras of the stereo system;

- detecting marks on the images, according to their coordinates a preliminary compensation of the displacement occurs using the perspective transformation;

- splitting the converted images into small parts (interrogating windows) for which the correlation function is calculated, the maximum of the function is the displacement of the parts relative to each other in images from different cameras;

- preliminary compensation of displacements and final calculation using cross-correlation gives two pairs of two-dimensional coordinates of one three-dimensional point in the images, triangulation by these coordinates allows to determine three-dimensional coordinates of surface points, in other words, restore the desired shape.

Image processing was carried out using the OpenCV library in the Python programming language. The cross-correlation processing algorithm is implemented in Python and allows iterative processing with a decrease of an interrogating window and postfiltering to increase the accuracy of the determined displacement. 
Fig. 3 shows an example of modeled images of a simulated aircraft wing. The surface size is $400 \times 200 \mathrm{~mm}^{2}$, it was divided into 1600 triangles. The parameters of the cameras corresponded to the real cameras used in the created system [6]. And their location relative to the wing corresponded to the approximate location of the system on an unmanned aerial vehicle. As a texture for the surface, we used a background pattern with randomly located dots and additional marks for preliminary image processing.

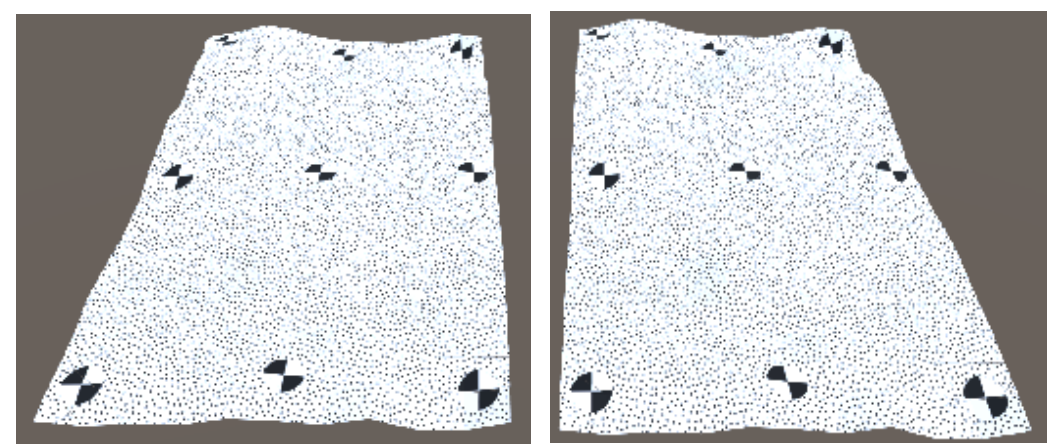

Fig. 3. Modeled images of a deformed surface of a simulated aircraft wing.

Fig. 4 shows an example of a vector field obtained by calculating the cross-correlation function. Processing was performed with the initial size of the interrogation window $32 \times 32$ pixels to a final size of $8 \times 8$ pixels in 3 iterations. The minimum value of the normalized correlation function at the maxima (the color of the arrows in Fig. 4) in the interrogation windows was at least 0.6 , which indicates the reliability of the calculation.

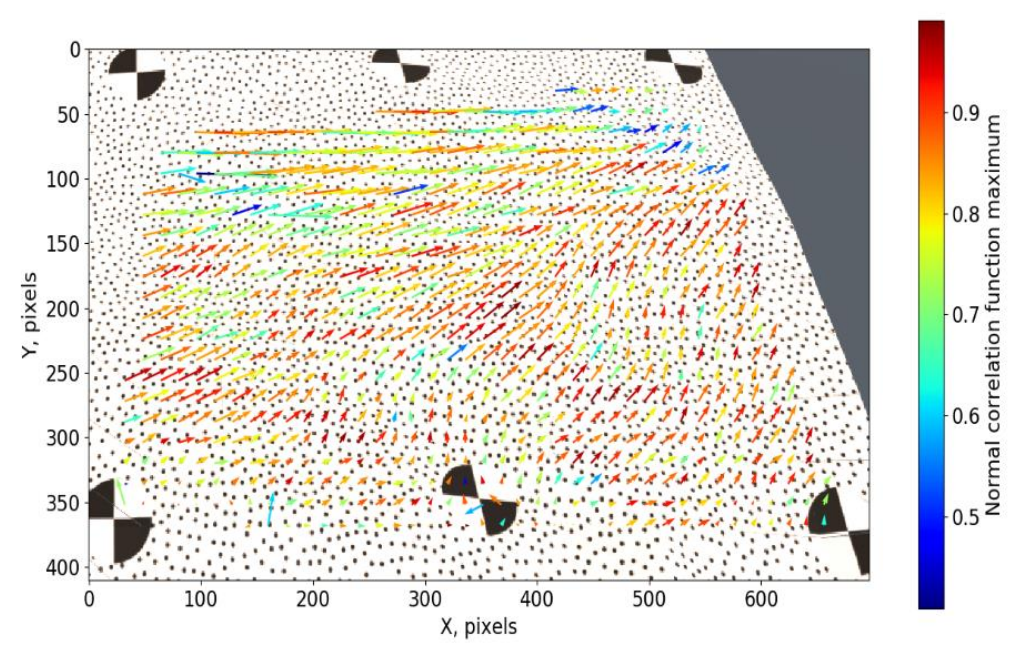

Fig. 4. The vector field obtained by calculating the cross-correlation function between two images in Fig. 3. 
After processing the modeled images, the results were compared with the given values of the surface shape during modeling. At the beginning, the coordinates of the surface points from Unity were transformed into the coordinate system of the camera stereo system. The coordinate system in Unity is left-handed, and in OpenCV right-handed. The cameras were also located at a certain distance from the origin and at an angle of $35^{\circ}$. These parameters also had to be taken into account when comparing the results. Further, the three-dimensional surface was interpolated for points specified in the modeling for comparison with points obtained during processing. The $\mathrm{x}$ and $\mathrm{y}$ coordinates were equal, the error along the $\mathrm{z}$ coordinate was calculated.

Fig. 5 shows an example of comparing processing results with set of points predefined during modeling. The standard deviation was less than $2 \mathrm{~mm}$ on the surface of 160x80 $\mathrm{mm}^{2}$, which indicates a high measurement error.

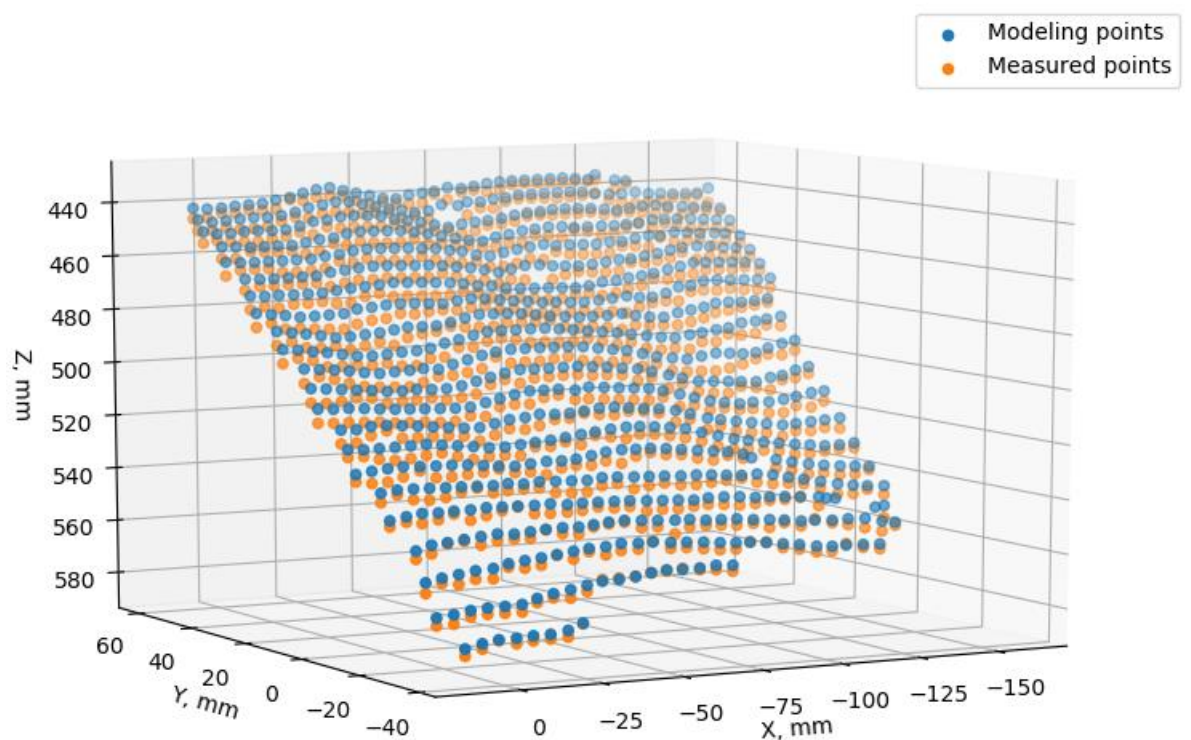

Fig. 5. Comparison of processing results with set of points predefined during modeling.

\section{Conclusions}

In the work, software was created for computer modeling of images of a deformed surface obtained using a stereo system. When modeling, it is possible to set both real camera parameters (external and internal parameters, including distortion coefficients), and parameters of the measured surface. A surface is defined using many connected triangles. Changing the parameters of their connection allows to bring the surface behavior closer to real physical objects, for example, the wing of an aircraft. Also, adding an additional random error into the predefined values during modeling allows to estimate the influence of the calibration accuracy of the stereo system of cameras on the measurement results. 
The developed methodology for computer modeling of images of the deformable surface allows to evaluate the accuracy of various algorithms for processing experimental images, as well as the influence of the parameters of the experimental setup on the error of the results.

\section{References}

1. Boden, F., Lawson, N., Jentink, H.W., Kompenhans, J. eds: Advanced In-Flight Measurement Techniques. Springer Berlin Heidelberg, Berlin, Heidelberg (2013). https://doi.org/10.1007/978-3-642-34738-2.

2. AIM, http://aim.dlr.de/site/index.php, last accessed 2020/06/29.

3. Kirmse, T.: Recalibration of a stereoscopic camera system for in-flight wing deformation measurements. Meas. Sci. Technol. 27, 054001 (2016). https://doi.org/10.1088/09570233/27/5/054001.

4. Kirmse, T., Maring, S., Ebel, P.-B., Schröder, A.: Fan blade deformation measurements on the DLR Airbus A320-ATRA by means of IPCT as part of the ground test campaign in the frame of the DLR-project SAMURAI. In: Dillmann, A., Heller, G., Krämer, E., Wagner, C., and Breitsamter, C. (eds.) New Results in Numerical and Experimental Fluid Mechanics X. pp. 629-638. Springer International Publishing, Cham (2016). https://doi.org/10.1007/9783-319-27279-5_55.

5. Boden, F., Jentink, H., Petit, C.: IPCT wing deformation measurements on a large transport aircraft. In: Boden, F., Lawson, N., Jentink, H.W., and Kompenhans, J. (eds.) Advanced inflight measurement techniques. pp. 93-115. Springer, Berlin, Heidelberg (2013). https://doi.org/10.1007/978-3-642-34738-2_7.

6. Poroykov A.Y., Surkov D.A., Ilina N.S., Lebedev S.V., Ul’yanov D.V., Lapitskiy K.M.: Development of the flight laboratory for research of aerodynamic surfaces deformation. J. Phys.: Conf. Ser. (2020). In print.

7. Petit C., Jentink H., Boden F., Kannemans H., Veermann H., Kirmse T.: Introducing a new measurement method for wing twist and bending. In: European Test \& Telemetry Conference 2009, pp. 1-6. (2009).

8. Poroikov, A.Yu.: Reconstruction of 3D Profile of a Deformed Metallic Plate by Means of the Image Pattern Correlation Technique. Meas Tech. 57, 390-395 (2014). https://doi.org/10.1007/s11018-014-0466-4.

9. Raffel, M., Willert, C.E., Scarano, F., Kähler, C.J., Wereley, S.T., Kompenhans, J.: Particle Image Velocimetry: A Practical Guide. Springer (2018).

10. Meier, G.: Computerized background-oriented schlieren. Exp Fluids. 33, 181-187 (2002). https://doi.org/10.1007/s00348-002-0450-7.

11. Poroykov A.Yu.: A system for determining the error in measuring the shape of a flexible deformable surface by means of the Image Pattern Correlation Technique. Herald of the Bauman Moscow State Tech. Univ., Instrum. Eng. 6, 28-39 (2017). https://doi.org/10.18698/0236-3933-2017-6-28-39.

12. Ivanova, Y.V., Poroykov, A.Yu.: Estimation of the measurement error of photogrammetric techniques by controlled flexible deformable surface. In: 2019 International Youth Conference on Radio Electronics, Electrical and Power Engineering (REEPE). pp. 1-4. IEEE, Moscow, Russia (2019). https://doi.org/10.1109/REEPE.2019.8708779.

13. Technologies, U.: Unity Real-Time Development Platform |3D, 2D VR \& AR Visualizations, https://unity.com/, last accessed 2020/06/29. 